\title{
Contribution of fat, sugar and salt to diets in the Pacific Islands: a systematic review
}

\author{
Joseph Alvin Santos 1,* , Briar McKenzie ${ }^{1}$, Kathy Trieu ${ }^{1}$, Sara Farnbach ${ }^{1,2}$, Claire Johnson ${ }^{\text {, }}$ \\ Jimaima Schultz ${ }^{3}$, Anne Marie Thow ${ }^{4}$, Wendy Snowdon ${ }^{5}$, Colin Bell ${ }^{5}$ and Jacqui Webster ${ }^{1}$ \\ ${ }^{1}$ The George Institute for Global Health, University of New South Wales, PO Box M201, Missenden Road, Sydney, \\ NSW 2052, Australia: ${ }^{2}$ National Drug and Alcohol Research Centre, University of New South Wales, Sydney, NSW, \\ Australia: ${ }^{3}$ Independent Nutrition Consultant, Suva, Fiji: ${ }^{4}$ Menzies Centre for Health Policy, School of Public Health, \\ The University of Sydney, Sydney, NSW, Australia: ${ }^{5}$ Global Obesity Centre, Deakin University, Geelong, VIC, Australia
}

Submitted 3 July 2018: Final revision received 26 October 2018: Accepted 9 November 2018: First published online 7 January 2019

\begin{abstract}
Objective: Pacific Island countries are experiencing a high burden of diet-related non-communicable diseases; and consumption of fat, sugar and salt are important modifiable risk factors contributing to this. The present study systematically reviewed and summarized available literature on dietary intakes of fat, sugar and salt in the Pacific Islands.

Design: Electronic databases (PubMed, Scopus, ScienceDirect and GlobalHealth) were searched from 2005 to January 2018. Grey literature was also searched and key stakeholders were consulted for additional information. Study eligibility was assessed by two authors and quality was evaluated using a modified tool for assessing dietary intake studies.

Results: Thirty-one studies were included, twenty-two contained information on fat, seventeen on sugar and fourteen on salt. Dietary assessment methods varied widely and six different outcome measures for fat, sugar and salt intake - absolute intake, household expenditure, percentage contribution to energy intake, sources, availability and dietary behaviours - were used. Absolute intake of fat ranged from $25.4 \mathrm{~g} / \mathrm{d}$ in Solomon Islands to $98.9 \mathrm{~g} / \mathrm{d}$ in Guam, while salt intake ranged from $5.6 \mathrm{~g} / \mathrm{d}$ in Kiribati to $10.3 \mathrm{~g} / \mathrm{d}$ in Fiji. Only Guam reported on absolute sugar intake $(47 \cdot 3 \mathrm{~g} / \mathrm{d})$. Peer-reviewed research studies used higher-quality dietary assessment methods, while reports from national surveys had better participation rates but mostly utilized indirect methods to quantify intake.

Conclusions: Despite the established and growing crisis of diet-related diseases in the Pacific, there is inadequate evidence about what Pacific Islanders are eating. Pacific Island countries need nutrition monitoring systems to fully understand the changing diets of Pacific Islanders and inform effective policy interventions.
\end{abstract}

\author{
Keywords \\ Fat intake \\ Sugar intake \\ Salt intake \\ Pacific Island countries
}

The Pacific Island region comprises a wide area of diverse nations that suffer from some of the highest rates of noncommunicable diseases (NCD) globally ${ }^{(1)}$. NCD, including CVD and diabetes, account for about $70 \%$ of all deaths in the region, most of which occur before the age of 60 years ${ }^{(2)}$. In fact, NCD are the primary cause of premature deaths in all Pacific Island countries ${ }^{(1)}$. Alarmingly, NCD rates are expected to increase given existing risk factors ${ }^{(1)}$ and the transition from traditional diets based on locally grown foods ${ }^{(3,4)}$ to modern diets characterized by imported foods and refined oils, sugar and confectionery, and processed meats ${ }^{(5,6)}$.
In 2013, the WHO recommended that all Member States develop policy measures for promoting a healthy diet, as part of efforts to reduce premature mortality from NCD by $25 \%$ by $2025^{(7)}$. Recommendations include policies to reduce population intakes of salt, SFA, trans-fats, and free and added sugars ${ }^{(7)}$. In Pacific Island countries, several initiatives have already been implemented such as changes in trade policy including import bans on carbonated soft drinks in Tokelau ${ }^{(8)}$; sales ban on mutton flaps in Fiji $^{(9)}$; import tariffs or excise taxes on sugary drinks in twelve Pacific Island countries ${ }^{(8,10)}$; regional food-based 
dietary guidelines ${ }^{(11)}$; and support for local agriculture and efforts to improve food security ${ }^{(12)}$. Evaluation of some of these efforts showed barriers to success including insufficient engagement from other sectors ${ }^{(9)}$, and there has been no evidence of significant decline in NCD rates in the region although stabilization of some risk factors is being seen $^{(13)}$.

A good nutrition monitoring system is important for developing and strengthening policies and actions and evaluating health interventions, especially in the Pacific where resources are scarce ${ }^{(14)}$. As such, data on current intakes of fat, sugar and salt are needed for setting realistic goals and plans for strategies to reduce consumption of these nutrients. These data are critical to lay the foundation of evidence-based interventions and for monitoring impact of interventions. While there have been reviews on dietary patterns and intakes in Pacific Island countries ${ }^{(3,15,16)}$, no reviews have been done in the last 10 years. Thus, we aimed to systematically review and synthesize recent literature on dietary intakes of Pacific Island adult populations, with a specific focus on fat, sugar and salt intake.

\section{Methods}

A search protocol was developed based on the Preferred Reporting Items for Systematic Reviews and Meta-Analyses (PRISMA) guidelines for systematic reviews. This included a defined search strategy, methods for data extraction and synthesis, and assessment of quality of the studies.

\section{Search protocol and databases}

A search for published literature was conducted using PubMed, Scopus, Science Direct and Global Health between 2005 and January 2018, limited to English language and human studies. The search strategy comprised three groups of search terms: nutrient terms (salt, sugar and fat), intake terms (eat, consumption, ingest and intake) and country terms (each of the twenty-two Pacific Island countries and areas, Pacific Islands, and Micronesia, Polynesia or Melanesia). A grey literature search was also performed using Google Scholar, Open Grey, Prism Survey and WHO databases, limited to the first ten pages of results. Key stakeholders, including experts, researchers and relevant organizations working to address diet-related issues in the Pacific Islands, were contacted to obtain information about unpublished documents or data sets.

\section{Study selection}

All search results were imported to EndNote X7.7.1 (Thomas Reuters, 2016) for title and abstract screening. Two authors (J.A.S. and B.M.) independently screened the titles and abstracts, and the full texts of potentially related articles were obtained and assessed further for eligibility. Disagreements at any stage of eligibility assessment (title and abstract screening, full-text review stage) were discussed by the two authors until agreement was reached. Studies not meeting the inclusion criteria as well as those not available electronically were excluded.

\section{Inclusion criteria}

Studies of any design were included if the primary or secondary outcomes provided information relevant to dietary patterns or preferences or intakes of fat, sugar and salt carried out on a general Pacific Island adult population (15-64 years of age). Countries of studies included were American Samoa, Commonwealth of the Northern Mariana Island (CNMI), Cook Islands, Federated States of Micronesia (FSM), Fiji, French Polynesia, Guam, Kiribati, Nauru, New Caledonia, Niue, Palau, Papua New Guinea (PNG), Pitcairn Island, Republic of the Marshall Islands (RMI), Samoa, Solomon Islands, Tokelau, Tonga, Tuvalu, Vanuatu, and Wallis and Futuna.

\section{Exclusion criteria}

Studies focusing on fortification, fishing, agriculture, disaster conditions, and those that included sick populations only were excluded. Studies specifically conducted in women who were lactating were excluded. Studies that only reported on data collected before 2005 were also excluded, regardless of the publication date.

\section{Data extraction and synthesis}

Data extraction was done by two authors (J.A.S. and B.M.) using a data extraction form designed for the present review. Information on study year, country of study, research design, type of participants and sample size, dietary assessment methods, and intake (e.g. description of intake or absolute intake, if reported) of fat, sugar and salt were extracted.

Where applicable, quality of the included studies was assessed by a modified tool for evaluating dietary intake studies $^{(17)}$. This tool was originally used to evaluate intake studies conducted among Aboriginal and Torres Strait Islanders in Australia. For the purpose of the present review, seven domains were employed, including: (i) involvement of Pacific Islanders in the study during conception, design or conduct of research; (ii) representativeness of sample of the underlying population; (iii) participation rate; (iv) reliability and validity of the dietary assessment tool; (v) quality of dietary assessment tool based on a set of criteria; (vi) accuracy of the food composition tables; and (vii) completeness and usability of results. More details on how each quality domain was scored are available in the online supplementary material, Supplemental Table 1. Two authors (J.A.S. and B.M.) assessed the quality of the studies and a third author (K.T.) was consulted for any disagreements in assessment. Each study was rated as low, high or unclear quality for each quality domain. 
A narrative synthesis of findings was based on all included studies. A descriptive approach was undertaken due to the heterogeneous nature of the reported outcomes.

\section{Results}

\section{Search results}

The search identified 1529 records (1472 from the peerreviewed and fifty-seven from the grey literature search and key stakeholders), of which 121 were considered potentially relevant (Fig. 1). Of these, seven full texts were not available and eighty-three were excluded. The reasons for excluding the studies were: no clear data on fat, sugar or salt intake ( $n$ 46); data collected before 2005 ( $n$ 10); not relevant ( $n$ 19); did not meet the age criteria $(n 7)$; and duplicate ( $n$ 1). Ultimately, thirty-one studies met the criteria.

\section{Description of the studies}

Study design varied across the included studies, ranging from cross-sectional ( $n$ 20) to pre-post intervention studies $(n 4)$, dietary modelling ( $n$ 4), use of secondary data ( $n$ 2) and case-control ( $n$ 1; Table 1$)$. Three studies ${ }^{(18-20)}$ included women only. Eleven studies used households as the unit of analysis ${ }^{(21-31)}$, fourteen reported the results at the individual level ${ }^{(18-20,32-40)}$ (two unpublished results: Fiji National Nutrition Survey 2017 and Vanuatu Ministry of Health 2017), while the rest described intake at a population level ${ }^{(41-46)}$. Sample sizes ranged from 150 to 4479 households and from thirty-four to 3220 individuals. Three studies ${ }^{(44-46)}$ included multiple Pacific Island countries, while the others involved a single country. Among the single-country studies, six were done in Samoa; three each in FSM, Solomon Islands and Vanuatu; two each in Fiji, Guam and Tonga; and one each in CNMI, Cook Islands, Nauru, New Caledonia, Palau, PNG and Tuvalu. No data

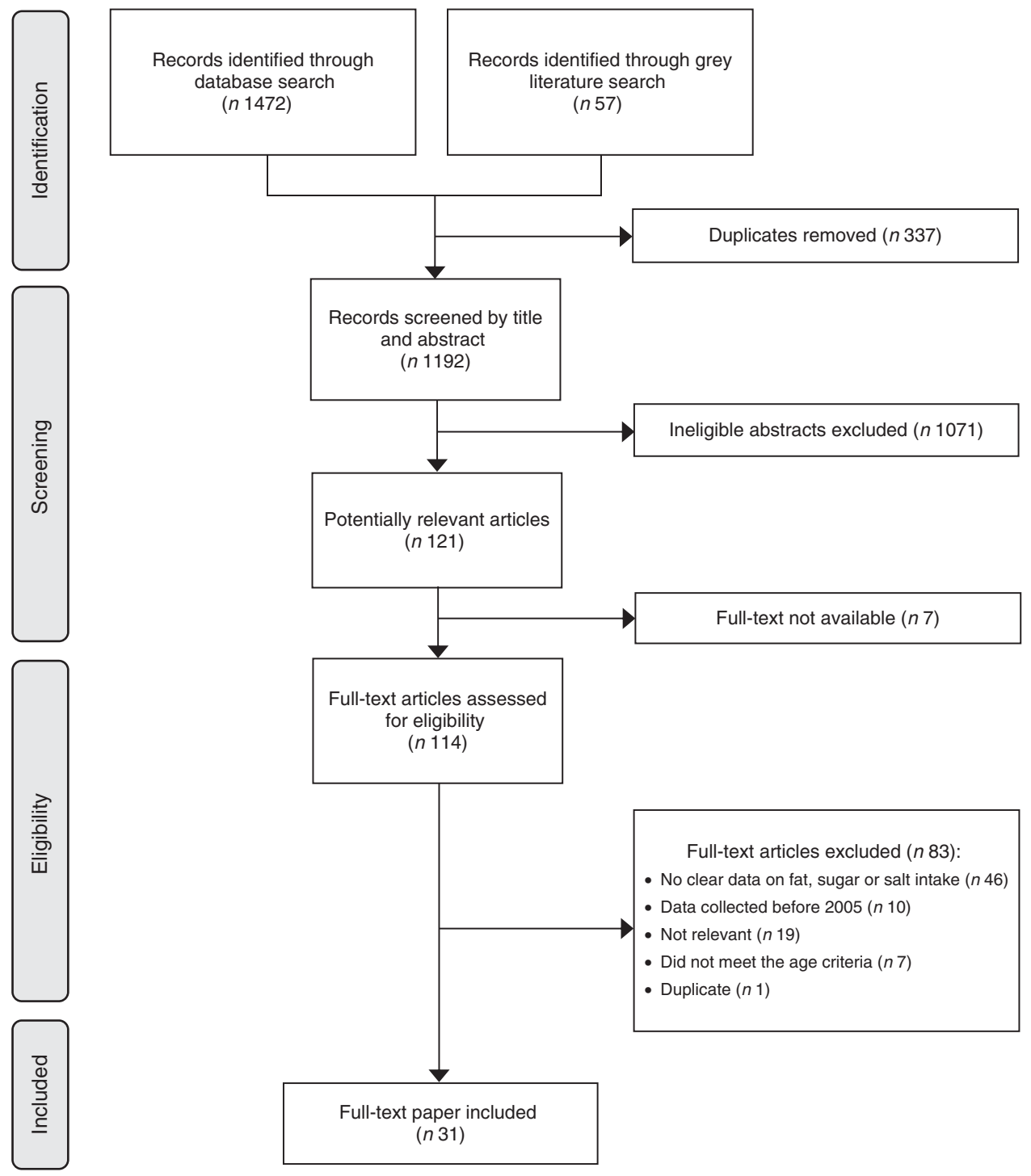

Fig. 1 Flowchart of studies included in the present systematic review on the contribution of fat, sugar and salt to diets in the Pacific Islands 
Table 1 Dietary study characteristics and reported data on fat, sugar and salt intake

\begin{tabular}{|c|c|c|c|c|c|c|}
\hline \multirow[b]{2}{*}{ Study, country } & \multirow{2}{*}{$\begin{array}{l}\text { Description, study } \\
\text { design }\end{array}$} & \multirow[b]{2}{*}{ Sample characteristics } & \multirow[b]{2}{*}{ Dietary assessment method } & \multicolumn{3}{|c|}{ Reported data on intake } \\
\hline & & & & Fat & Sugar & Salt \\
\hline $\begin{array}{l}\text { Dela Cruz and Cash } \\
(2016)^{(32)} \\
\text { CNMI }\end{array}$ & $\begin{array}{l}\text { Hybrid NCD and risk } \\
\text { factor household } \\
\text { survey } \\
\text { Cross-sectional }\end{array}$ & $\begin{array}{l}\text { Household survey; adults aged } \\
18 \text { years or above; } n 1091\end{array}$ & $\begin{array}{l}\text { NCD risk factor } \\
\text { questionnaire }\end{array}$ & & $\mathrm{BE}$ & $\mathrm{BE}$ \\
\hline $\begin{array}{l}\text { Statistics Office } \\
(2007)^{(21)} \\
\text { Cook Islands }\end{array}$ & $\begin{array}{l}\text { Household } \\
\text { Expenditure Survey } \\
\text { Cross-sectional }\end{array}$ & $\begin{array}{l}773 \text { households from three } \\
\text { regions in Cook Islands }\end{array}$ & $\begin{array}{l}\text { Two-week food diary and } \\
\text { expenditure }\end{array}$ & EX & EX & \\
\hline $\begin{array}{l}\text { Statistics Division } \\
(2014)^{(22)} \\
\text { FSM }\end{array}$ & $\begin{array}{c}\text { HIES } \\
\text { Cross-sectional }\end{array}$ & 1988 households & $\begin{array}{l}\text { Two-week food diary and } \\
\text { expenditure }\end{array}$ & EX & EX & \\
\hline $\begin{array}{l}\text { Hanson et al. } \\
(2011)^{(33)} \\
\text { FSM }\end{array}$ & $\begin{array}{l}\text { Nutrition intervention } \\
\text { Pre-post intervention } \\
\text { study }\end{array}$ & $\begin{array}{l}\text { One adult from each } \\
\text { household; seventy-five } \\
\text { households at baseline and } \\
\text { sixty-eight at follow-up }\end{array}$ & $\begin{array}{c}7 \mathrm{~d} \text { Helen Keller International } \\
\text { FFQ }\end{array}$ & BE & BE & $\mathrm{BE}$ \\
\hline $\begin{array}{l}\text { Kaufer et al. } \\
(2010)^{(18)} \\
\text { FSM }\end{array}$ & $\begin{array}{c}\text { Food-based } \\
\text { intervention } \\
\text { Pre-post intervention } \\
\text { study }\end{array}$ & $\begin{array}{l}\text { One adult woman from each } \\
\text { household; forty households } \\
\text { within a Pohnpeian community }\end{array}$ & $\begin{array}{l}7 \mathrm{~d} \text { FFQ; two non- } \\
\text { consecutive } 24 \mathrm{H}-\mathrm{FR} \text { and an } \\
\text { attitudes questionnaire }\end{array}$ & $\mathrm{Al}, \mathrm{PC}, \mathrm{BE}$ & $\mathrm{BE}$ & \\
\hline $\begin{array}{l}\text { Fiji National Nutrition } \\
\text { Survey } 2017 \\
\text { (unpublished } \\
\text { results) } \\
\text { Fiji }\end{array}$ & $\begin{array}{l}\text { National Nutrition } \\
\quad \text { Survey } \\
\text { Cross-sectional }\end{array}$ & 3220 adults & $24 \mathrm{H}-\mathrm{FR}$ & Al, MS & & Al, MS \\
\hline $\begin{array}{l}\text { Pillay et al. }(2017)^{(34)} \\
\text { Fiji }\end{array}$ & $\begin{array}{c}\text { Salt reduction } \\
\text { intervention } \\
\text { Pre-post intervention } \\
\text { study }\end{array}$ & $\begin{array}{l}\text { Adults aged } 25-64 \text { years; } n \\
169 \text { at baseline and } n 272 \text { at } \\
\text { follow-up }\end{array}$ & $24 \mathrm{H}-\mathrm{UNa}$ & & & Al \\
\hline $\begin{array}{l}\text { Leon-Guerrero et al. } \\
(2015)^{(35)} \\
\text { Guam }^{(30)}\end{array}$ & $\begin{array}{l}\text { Validity and reliability } \\
\text { study } \\
\text { Cross-sectional }\end{array}$ & $\begin{array}{c}\text { Adults aged } 18-61 \text { years; } n \\
109\end{array}$ & $\begin{array}{l}\text { Quantitative FFQ validated } \\
\text { by } 2 d \text { food record }\end{array}$ & Al & & Al \\
\hline $\begin{array}{l}\text { Paulino et al. } \\
\qquad(2011)^{(19)} \\
\text { Guam }\end{array}$ & $\begin{array}{l}\text { Comparative study of } \\
\text { intake between feast } \\
\text { and non-feast days } \\
\text { Cross-sectional }\end{array}$ & $\begin{array}{l}\text { Women aged } 40 \text { years and } \\
\text { above living in Guam; } n 25 \\
\text { Chamorro and } n 24 \text { Filipino }\end{array}$ & $\begin{array}{l}24 \mathrm{H}-\mathrm{FR} \text { of a previous feast } \\
\text { day and a non-feast day }\end{array}$ & PC & Al & \\
\hline $\begin{array}{l}\text { Bureau of Statistics } \\
(2014)^{(23)} \\
\text { Nauru }\end{array}$ & $\begin{array}{c}\text { HIES } \\
\text { Cross-sectional }\end{array}$ & 515 households & $\begin{array}{l}\text { Two-week food diary and } \\
\text { expenditure }\end{array}$ & EX & $\mathrm{EX}$ & \\
\hline $\begin{array}{l}\text { Baudchon }(2009)^{(24)} \\
\text { New Caledonia }\end{array}$ & $\begin{array}{l}\text { Household } \\
\text { Consumption Survey } \\
\text { Cross-sectional }\end{array}$ & 3704 households & $\begin{array}{l}\text { Record of expenses and } \\
\text { resources of households }\end{array}$ & EX & EX & \\
\hline $\begin{array}{l}\text { Office of Planning and } \\
\text { Statistics }(2014)^{(25)} \\
\text { Palau }\end{array}$ & $\begin{array}{c}\text { HIES } \\
\text { Cross-sectional }\end{array}$ & 1145 households & $\begin{array}{l}\text { Two-week food diary and } \\
\text { expenditure }\end{array}$ & EX & EX & \\
\hline $\begin{array}{l}\text { Temple et al. } \\
(2010)^{(26)} \\
\text { PNG }\end{array}$ & $\begin{array}{l}\text { Salt iodization and } \\
\text { consumption survey } \\
\text { Cross-sectional }\end{array}$ & $\begin{array}{l}150 \text { households in Moresby } \\
\text { North East and Moresby South }\end{array}$ & Salt weighing & & & Al \\
\hline $\begin{array}{l}\text { Trieu et al. }(2017)^{(36)} \\
\text { Samoa }\end{array}$ & $\begin{array}{c}\text { Salt reduction } \\
\text { intervention } \\
\text { Pre-post intervention } \\
\text { study }\end{array}$ & $\begin{array}{l}\text { Adults aged } 18-64 \text { years; } n \\
234 \text { at baseline and } n 479 \text { at } \\
\text { follow-up }\end{array}$ & $24 \mathrm{H}-\mathrm{UNa}$ & & & $\mathrm{Al}$ \\
\hline $\begin{array}{l}\text { Martyn et al. } \\
\quad(2017)^{(41)} \\
\text { Samoa }\end{array}$ & $\begin{array}{l}\text { Identification of } \\
\text { household factors } \\
\text { associated with } \\
\text { nutrition } \\
\text { Modelling }\end{array}$ & $\begin{array}{l}\text { Data from HIES: } 16356 \\
\text { individuals and } 2791 \\
\text { households }\end{array}$ & $\begin{array}{l}\text { Data on household food } \\
\text { expenditure from HIES }\end{array}$ & $\mathrm{AV}, \mathrm{MS}$ & PC & $\mathrm{AV}, \mathrm{MS}$ \\
\hline
\end{tabular}




\begin{tabular}{|c|c|c|c|c|c|c|}
\hline \multirow[b]{2}{*}{ Study, country } & \multirow{2}{*}{$\begin{array}{l}\text { Description, study } \\
\text { design }\end{array}$} & \multirow[b]{2}{*}{ Sample characteristics } & \multirow[b]{2}{*}{ Dietary assessment method } & \multicolumn{3}{|c|}{ Reported data on intake } \\
\hline & & & & Fat & Sugar & Salt \\
\hline $\begin{array}{l}\text { Webster et al. } \\
\quad(2016)^{(37)} \\
\text { Samoa }\end{array}$ & $\begin{array}{l}\text { Assessment of salt } \\
\text { intake } \\
\text { Cross-sectional }\end{array}$ & $\begin{array}{c}293 \text { individuals aged } 18-64 \\
\text { years }\end{array}$ & $\begin{array}{c}24 \mathrm{H}-\mathrm{UNa} \text { and behaviours } \\
\text { questionnaire }\end{array}$ & & & $\mathrm{Al}, \mathrm{BE}$ \\
\hline $\begin{array}{l}\text { Samoa Bureau of } \\
\text { Statistics (2016) } \\
\text { Samoa }\end{array}$ & $\begin{array}{c}\text { HIES } \\
\text { Cross-sectional }\end{array}$ & 2348 households & $\begin{array}{c}\text { Two-week food diary and } \\
\text { expenditure }\end{array}$ & EX & EX & \\
\hline $\begin{array}{l}\text { Land et al. }(2016)^{(20)} \\
\text { Samoa }\end{array}$ & $\begin{array}{l}\text { Assessment of salt } \\
\text { intake and iodine } \\
\text { status } \\
\text { Cross-sectional }\end{array}$ & 119 women aged $18-45$ years & $24 \mathrm{H}-\mathrm{UNa}$ & & & $\mathrm{Al}$ \\
\hline $\begin{array}{l}\text { Seiden et al. } \\
(2012)^{(42)} \\
\text { Samoa }\end{array}$ & $\begin{array}{c}\text { Trends in food } \\
\text { availability and pricing } \\
\text { Secondary analysis of } \\
\text { data }\end{array}$ & National-level data & $\begin{array}{l}\text { Data from annual food } \\
\text { balance sheets }\end{array}$ & $\mathrm{AV}, \mathrm{MS}$ & & \\
\hline $\begin{array}{l}\text { Tsuchiya et al. } \\
(2017)^{(38)_{*}} \\
\text { Solomon Islands }\end{array}$ & $\begin{array}{l}\text { Assessment of factors } \\
\text { associated with obesity } \\
\text { Case-control }\end{array}$ & $\begin{array}{c}\text { Fifty-seven controls aged } 20 \\
\text { years or above }\end{array}$ & 24H-FR & $\mathrm{Al}, \mathrm{PC}, \mathrm{BE}$ & & \\
\hline $\begin{array}{l}\text { Solomon Islands } \\
\text { National Statistics } \\
\text { Office }(2015)^{(28)} \\
\text { Solomon Islands }\end{array}$ & $\begin{array}{c}\text { HIES } \\
\text { Cross-sectional }\end{array}$ & 4479 households & $\begin{array}{c}\text { Two-week food diary and } \\
\text { expenditure }\end{array}$ & EX & EX & \\
\hline $\begin{array}{l}\text { Aswani and } \\
\text { Furusawa } \\
(2007)^{(39)} \\
\text { Solomon Islands }\end{array}$ & $\begin{array}{l}\text { Comparative study } \\
\text { between marine } \\
\text { protected areas and } \\
\text { not } \\
\text { Cross-sectional }\end{array}$ & $\begin{array}{c}\text { Twenty households from six } \\
\text { villages; participants aged } 15 \\
\text { years or above }\end{array}$ & 24H-FR & $\mathrm{Al}$ & & \\
\hline $\begin{array}{l}\text { Konishi et al. } \\
(2011)^{(40)} \\
\text { Tonga }\end{array}$ & $\begin{array}{l}\text { Assessment of nutrient } \\
\text { intake and food sources } \\
\text { Cross-sectional }\end{array}$ & $\begin{array}{l}\text { Thirty-four adults aged } 40-59 \\
\text { years }\end{array}$ & $\begin{array}{l}\text { Two seven-consecutive-day } \\
24 \mathrm{H}-\mathrm{FR} \text { in two different } \\
\text { seasons; food weighing }\end{array}$ & Al, MS, PC & & \\
\hline $\begin{array}{l}\text { Statistics } \\
\text { Department } \\
(2010)^{(29)} \\
\text { Tonga }\end{array}$ & $\begin{array}{c}\text { HIES } \\
\text { Cross-sectional }\end{array}$ & 1983 households & $\begin{array}{l}\text { Two-week food diary and } \\
\text { expenditure }\end{array}$ & EX & EX & \\
\hline $\begin{array}{l}\text { Central Statistics } \\
\text { Division }(2010)^{(30)} \\
\text { Tuvalu }\end{array}$ & $\begin{array}{c}\text { HIES } \\
\text { Cross-sectional }\end{array}$ & 541 households & $\begin{array}{l}\text { Two-week food diary and } \\
\text { expenditure }\end{array}$ & EX & EX & \\
\hline $\begin{array}{l}\text { Vanuatu Ministry of } \\
\text { Health } 2017 \\
\text { (unpublished } \\
\text { results) } \\
\text { Vanuatu }\end{array}$ & $\begin{array}{l}\text { Assessment of salt } \\
\text { intake } \\
\text { Cross-sectional }\end{array}$ & $\begin{array}{l}\text { Adults aged } 18-69 \text { years; } n \\
483\end{array}$ & $\begin{array}{l}\text { Spot urine sample and } \\
\text { behaviours questionnaire }\end{array}$ & & & $\mathrm{Al}, \mathrm{BE}$ \\
\hline $\begin{array}{l}\text { Martyn et al. } \\
(2015)^{(43)} \\
\text { Vanuatu }\end{array}$ & $\begin{array}{l}\text { Identification of } \\
\text { household factors } \\
\text { associated with } \\
\text { nutrition } \\
\text { Modelling }\end{array}$ & $\begin{array}{l}\text { Data from HIES: } 3975 \\
\text { households from thirty islands }\end{array}$ & $\begin{array}{l}\text { Data on household food } \\
\text { expenditure from HIES }\end{array}$ & $\mathrm{Al}, \mathrm{MS}$ & EX, PC & Al, MS \\
\hline $\begin{array}{l}\text { Vanuatu National } \\
\text { Statistics Office } \\
(2012)^{(31)} \\
\text { Vanuatu }\end{array}$ & $\begin{array}{c}\text { HIES } \\
\text { Cross-sectional }\end{array}$ & 3975 households & $\begin{array}{l}\text { Two-week food diary and } \\
\text { expenditure }\end{array}$ & EX & EX & EX \\
\hline $\begin{array}{l}\text { Estime et al. } \\
(2014)^{(44)} \\
\text { Multiple countries }\end{array}$ & $\begin{array}{l}\text { Examination of links } \\
\text { between trade and } \\
\text { NCD } \\
\text { Secondary analysis of } \\
\text { data }\end{array}$ & $\begin{array}{l}\text { Data from five countries: } \\
\text { Samoa, } n \text { 2012; Solomon } \\
\text { Islands, } n \text { 3822; Tonga, } n \\
\text { 1640; Kiribati, } n \text { 1161; } \\
\text { Vanuatu, } n \text { not reported } \\
\text { (households) }\end{array}$ & $\begin{array}{l}\text { Secondary analysis of } \\
\text { household-level food } \\
\text { expenditure and } \\
\text { consumption data }\end{array}$ & & EX, PC & \\
\hline
\end{tabular}




\begin{tabular}{|c|c|c|c|c|c|c|}
\hline \multirow[b]{2}{*}{ Study, country } & \multirow{2}{*}{$\begin{array}{l}\text { Description, study } \\
\text { design }\end{array}$} & \multirow[b]{2}{*}{ Sample characteristics } & \multirow[b]{2}{*}{ Dietary assessment method } & \multicolumn{3}{|c|}{ Reported data on intake } \\
\hline & & & & Fat & Sugar & Salt \\
\hline $\begin{array}{l}\text { Micha et al. } \\
(2014)^{(46)} \\
\text { Multiple countries }\end{array}$ & $\begin{array}{l}\text { Estimation of global, } \\
\text { regional and national } \\
\text { dietary fat intake } \\
\text { Modelling }\end{array}$ & $\begin{array}{l}\text { Data from } 266 \text { surveys in } \\
\text { adults from } 113 \text { countries }\end{array}$ & $\begin{array}{c}\text { Data from previous surveys: } \\
\text { Bayesian hierarchical } \\
\text { modelling }\end{array}$ & PC & & \\
\hline $\begin{array}{l}\text { Powles et al. } \\
(2013)^{(45)} \\
\text { Multiple countries }\end{array}$ & $\begin{array}{l}\text { Estimation of global, } \\
\text { regional and national } \\
\text { salt intake } \\
\text { Modelling }\end{array}$ & $\begin{array}{l}\text { Data from } 142 \text { surveys of } 24 \mathrm{~h} \\
\text { urinary } \mathrm{Na} \text { and } 103 \text { dietary } \mathrm{Na} \\
\text { from sixty-six countries }\end{array}$ & $\begin{array}{c}\text { Data from previous surveys; } \\
\text { Bayesian hierarchical } \\
\text { modelling }\end{array}$ & & & Al \\
\hline
\end{tabular}

CNMI, Commonwealth of the Northern Mariana Islands; FSM, Federated States of Micronesia; PNG, Papua New Guinea; NCD, non-communicable disease; HIES, Household Income and Expenditure Survey; $24 \mathrm{H}-\mathrm{FR}, 24 \mathrm{~h}$ food recall; $24 \mathrm{H}-\mathrm{UNa}, 24 \mathrm{~h}$ urine collection; EX, household expenditure; BE, dietary behaviour; $\mathrm{Al}$, absolute intake; PC, percentage contribution to energy intake; MS, main sources; AV, availability.

*Only estimates for the control group were included.

were obtained from six countries: American Samoa, French Polynesia, Niue, Pitcairn Island, Tokelau, and Wallis and Futuna. Lastly, more than half ( $n$ 17) of the studies were from the grey literature and these were mainly government surveys.

\section{Quality of studies}

A summary of the quality assessment of studies included in the current review is presented in Fig. 2. Only two studies ${ }^{(20,37)}$ were of high quality across all domains. The number of studies where quality was unclear or low was similar for peer-reviewed and grey literature in four domains: involvement of Pacific Islanders in the study ( $n 1$ $v$. 2); representativeness of the sample ( $n 8 v$.9); quality of dietary assessment tool ( $n 1 v .2)$; and completeness and usability of results (both $n 0$ ). The number of studies where quality was unclear or low based on participation rate was higher for peer-reviewed compared with grey literature ( $n 7$ $v$. 1), while the opposite was true for reliability and validity of the dietary assessment tool ( $n 4 v .15)$.

\section{Dietary assessment method and type of outcome data reported}

Use of Household Income and Expenditure Survey (HIES) data, comprised of a two-week diary and records of expenditure on food, was the most common method for assessing dietary intake in studies ( $n$ 10), followed by $24 \mathrm{~h}$ food recall ( $n$ 6 ) and $24 \mathrm{~h}$ urine collection $(n 4)$ and diet questionnaire $(n 4)$. Other assessment methods employed were FFQ, dietary modelling, review of data from past surveys, food weighing, spot urine collection and food records. Five studies ${ }^{(18,35,37,40)}$ (one unpublished results: Vanuatu Ministry of Health 2017) used more than one method to measure dietary intake. Twenty-two studies published information on fat intake, seventeen on sugar intake and fourteen on salt intake. Overall, six types of outcomes regarding intake of fat, sugar or salt were reported: (i) absolute intake; (ii) household expenditure; (iii) percentage contribution to total energy intake; (iv) main sources; (v) availability (i.e. foods available for consumption, per capita); and (vi) dietary behaviours.

Absolute intake, main sources and percentage contribution of fat, sugar and salt to total energy intake were commonly measured by $24 \mathrm{~h}$ food recall, FFQ or dietary modelling. To measure salt intake, four studies from two countries (one in Fiji and three in Samoa) ${ }^{(20,34,36,37)}$ utilized the gold standard $24 \mathrm{~h}$ urine collection, while others employed spot urine collection and salt weighing. Household expenditure was measured using HIES data. Information on availability or access was either from secondary data or dietary modelling, while behaviours were assessed through $24 \mathrm{~h}$ food recall, FFQ or diet questionnaire.

\section{Fat, sugar and salt intake}

\section{Absolute intake}

Results for each outcome measure are presented in Table 2 (fat intake), Table 3 (sugar intake) and Table 4 (salt intake). Information on absolute intake of fat and salt was available in six and ten countries, respectively, while only one country (Guam) ${ }^{(19)}$ had data on absolute intake of added sugar $(47 \mathrm{~g} / \mathrm{d})$. Compared with total fat intake in Solomon Islands $(25 \mathrm{~g} / \mathrm{d})^{(38)}$, intake in other countries was high (mean intake of $99 \mathrm{~g} / \mathrm{d}$ in Guam ${ }^{(35)}, 97 \mathrm{~g} / \mathrm{d}$ in Fiji (Fiji National Nutrition Survey 2017, unpublished results), $75 \mathrm{~g} / \mathrm{d}$ in Vanuatu ${ }^{(43)}, 63 \mathrm{~g} / \mathrm{d}$ in $\mathrm{FSM}^{(18)}, 107 \mathrm{~g} / \mathrm{d}$ for males and $88 \mathrm{~g} / \mathrm{d}$ for females in Tonga $\left.{ }^{(40)}\right)$.

Of the ten countries with data on absolute salt intake, only two (Fiji and Samoa) were based upon $24 \mathrm{~h}$ urine collection. Four countries (Fiji, PNG, Samoa and Vanuatu) had multiple data points, but differences in study design, assessment methods and sampling did not allow for assessment of change over time. Five countries (FSM, Kiribati, RMI, Solomon Islands and Tonga) had salt intake estimates solely based from modelling ${ }^{(45)}$. The most recent data on intake of fat, sugar and salt, and the dietary assessment method used, are shown in Table 5. 


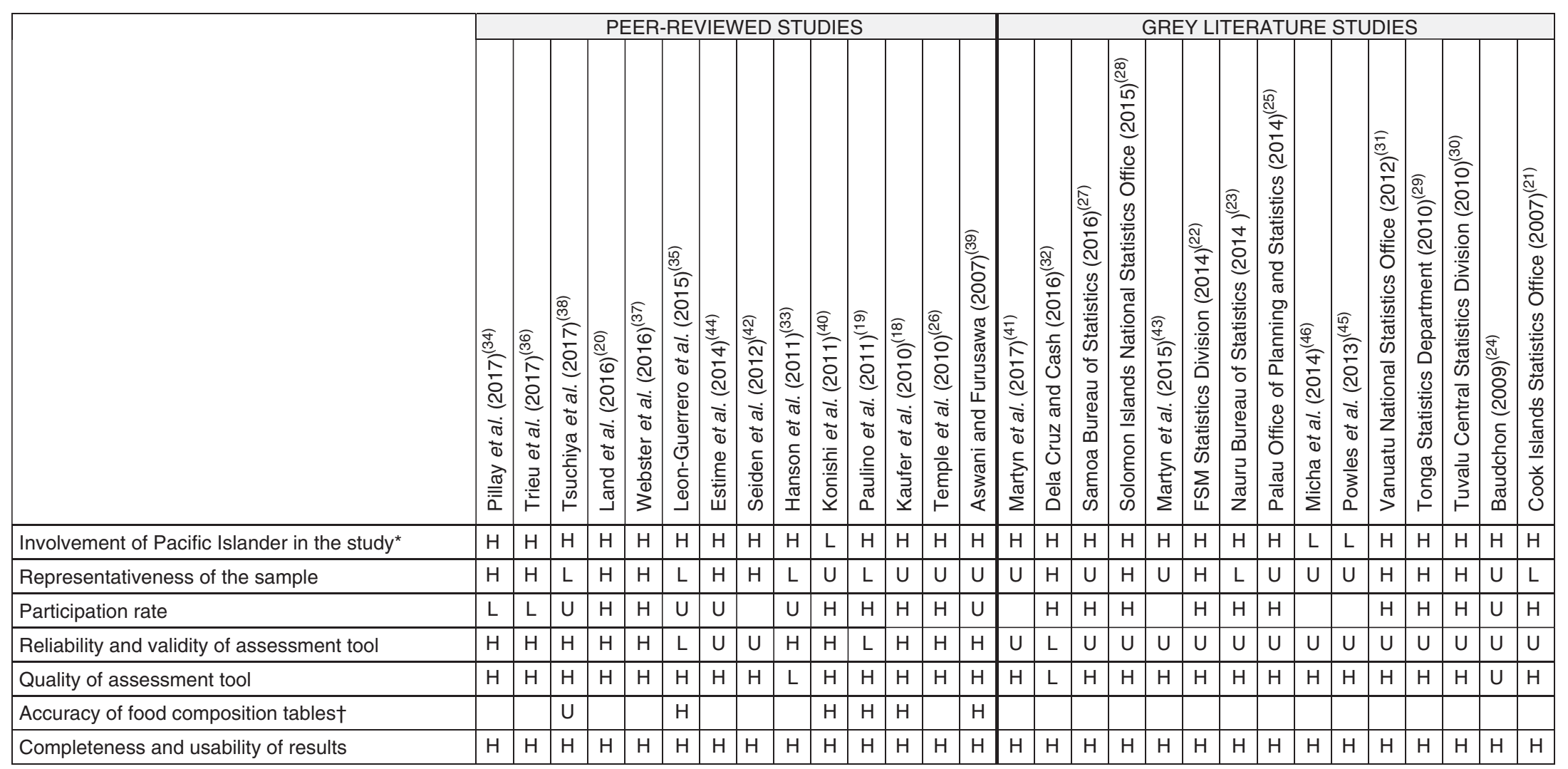

Fig. 2 Summary of quality assessment $(\mathrm{H}$, high quality; $\mathrm{U}$, unclear quality; L, low quality; blank, not applicable) of studies included in the present systematic review on the contribution of fat, sugar and salt to diets in the Pacific Islands (note, quality assessment of two unpublished results was not completed: Fiji National Nutrition Survey 2017 and Vanuatu Ministry of Health 2017). * Refers to involvement during conception, design, conduct of research, including pre-testing and data collection, and distribution of results. †Assessed based on the appropriateness of the food composition database used to analyse the data, and whether a second person checked the linking of foods to the food composition table (FSM, Federated States of Micronesia) 
Table 2 Fat intake by type of outcome reported and country

\begin{tabular}{|c|c|c|}
\hline Type of intake data & $\begin{array}{l}\text { Countries } \\
\text { with data }\end{array}$ & Description of intake \\
\hline \multirow[t]{7}{*}{ Absolute intake* } & FSM & $\begin{array}{l}\text { Kaufer, } 2010 \text { (24H-FR; adult women): } 2005 \text { (pre-intervention) } 61.3 \mathrm{~g} / \mathrm{d} ; 2007 \text { (post-intervention) } \\
62.6 \mathrm{~g} / \mathrm{d}\end{array}$ \\
\hline & Fiji & National Nutrition Survey, 2017 (24H-FR; adults): 97.1 (95\% Cl 96.0, 98.2) g/d \\
\hline & Guam & $\begin{array}{l}\text { Leon-Guerrero, } 2015 \text { (FFQ; adults aged 18-61 years): total fat } 98.9 \text { (sD } 67.7) \mathrm{g} / \mathrm{d} \text {; saturated fat } \\
33.0 \text { (sD 24.0) g/d; MUFA } 35.7 \text { (SD 24.6) g/d; PUFA (SD 14.8) } 21.4 \mathrm{~g} / \mathrm{d}\end{array}$ \\
\hline & Solomon & Tsuchiya, 2017 (24H-FR; controls aged $\geq 20$ years): 25.4 (sD 13.2) g/d \\
\hline & Islands & $\begin{array}{l}\text { Aswani, } 2007 \text { (24H-FR; adults aged } \geq 15 \text { years): males - Kozou } 26.4 \text { (sD 4.6) g/d; Nusa Hope } 25.0 \\
\text { (sD 2.7) g/d; Baraulu } 33.7 \text { (sD 4.3) g/d; Olive } 25.5 \text { (sD 3.0) g/d; Dunde } 41.6 \text { (sD 3.8) g/d; Nusa } \\
\text { Banga } 19.7 \text { (sD 4.0) g/d; females - Kozou } 16.4 \text { (sD 3.6) g/d; Nusa Hope } 35.7 \text { (sD 5.0) g/d; Baraulu } \\
31.2 \text { (sD 2.8) g/d; Olive 25.0 (sD 2.8) g/d; Dunde } 42.8 \text { (sD 6.8) g/d; Nusa Banga 25.6 (sD 14.5) g/d }\end{array}$ \\
\hline & Tonga & $\begin{array}{l}\text { Konishi, } 2011 \text { ( } 24 \mathrm{H}-\mathrm{FR} \text {; adults aged } 40-59 \text { years; median intake): males } 107.0 \mathrm{~g} / \mathrm{d} \text {; females } \\
\quad 88.0 \mathrm{~g} / \mathrm{d}\end{array}$ \\
\hline & Vanuatu & Martyn, 2015 (modelling): $75.0 \mathrm{~g} / \mathrm{d}$; rural $73.0 \mathrm{~g} / \mathrm{d}$; urban $81.0 \mathrm{~g} / \mathrm{d}$ \\
\hline \multirow[t]{10}{*}{ Household expenditure } & Cook Islands & 2007: $3.4 \%$ \\
\hline & FSM & 2014: $0.9 \%$ \\
\hline & Nauru & 2014: $2.0 \%$ \\
\hline & $\begin{array}{l}\text { New } \\
\text { Caledonia }\end{array}$ & 2009: $1.5 \%$ \\
\hline & Palau & 2014: $2.0 \%$ \\
\hline & Samoa & 2016: $0.9 \%$ \\
\hline & $\begin{array}{l}\text { Solomon } \\
\text { Islands }\end{array}$ & 2015: $0.7 \%$ \\
\hline & Tonga & 2010: $3.1 \%$ \\
\hline & Tuvalu & 2010: $3 \cdot 2 \%$ \\
\hline & Vanuatu & 2012: $0.01 \%$ \\
\hline \multirow[t]{5}{*}{ Main sources } & Fiji & $\begin{array}{l}\text { National Nutrition Survey, } 2017 \text { ( } 24 \mathrm{H}-\mathrm{FR} \text {; adults): fish and seafood (fresh, canned, mixed food) } \\
22.3 \% \text {, animal-source foods (poultry, mixed food, fresh meat and sausages) } 18.8 \% \text {, wheat flour } \\
\text { products (crackers, roti, instant noodles, sweets) } 15.1 \% \text {, vegetable fat } 8.6 \% \text {, coconut products } \\
8.4 \% \text {, starchy staples } 5.4 \%\end{array}$ \\
\hline & Samoa & $\begin{array}{l}\text { Martyn, } 2017 \text { (modelling): coconuts (popo) } 19.0 \% \text {, chicken pieces } 13.0 \% \text {, canned mackerel } \\
8.0 \% \text {, cooking oil } 6.0 \%\end{array}$ \\
\hline & & $\begin{array}{l}\text { Seiden, } 2012 \text { (secondary analysis): coconuts and copra, vegetable oils, bovine meat, mutton and } \\
\text { goat meat, pig meat, poultry meat }\end{array}$ \\
\hline & Tonga & $\begin{array}{l}\text { Konishi, } 2011 \text { ( } 24 \mathrm{H}-\mathrm{FR} \text {; adults aged } 40-59 \text { years): imported foods contributed } 65 \% \text { of total daily } \\
\text { fat intake }\end{array}$ \\
\hline & Vanuatu & $\begin{array}{l}\text { Martyn, } 2015 \text { (modelling): coconut or copra, cooking oil, peanuts, cabin biscuits, fresh beef, } \\
\text { chicken }\end{array}$ \\
\hline \multirow{13}{*}{$\begin{array}{l}\text { Percentage contribution } \\
\text { to energy } \dagger\end{array}$} & FSM & Micha, 2014 (modelling; saturated fat): $22 \cdot 9 \%$ \\
\hline & & $\begin{array}{l}\text { Kaufer, } 2010 \text { (24H-FR; adult women): } 2005 \text { (pre-intervention) 23.3\%; } 2007 \text { (post-intervention) } \\
\text { 26.2\% }\end{array}$ \\
\hline & Fiji & Micha, 2014 (modelling; saturated fat): $23 \cdot 8 \%$ \\
\hline & Guam & $\begin{array}{l}\text { Paulino, } 2011 \text { (24H-FR; women aged } \geq 40 \text { years): total fat }- \text { feast day } 34.1 \text { (SD } 7.8 \text { ) \%; non-feast } \\
\text { day } 27.5 \text { (SD } 9.6 \text { ) \%; saturated fat - feast day } 11.4 \text { (SD } 4.7) \% \text {; non-feast day } 7.9 \text { (SD } 3.4 \text { ) \% }\end{array}$ \\
\hline & Kiribati & Micha, 2014 (modelling; saturated fat): $27.0 \%$ \\
\hline & PNG & Micha, 2014 (modelling; saturated fat): $23.2 \%$ \\
\hline & RMI & Micha, 2014 (modelling; saturated fat): $22.9 \%$ \\
\hline & Samoa & Micha, 2014 (modelling; saturated fat): $27.5 \%$ \\
\hline & Solomon & Tsuchiya, 2017 (24H-FR; controls aged $\geq 20$ years): 14.5 (sD $6 \cdot 7) \%$ \\
\hline & Islands & Micha, 2014 (modelling; saturated fat): $23.9 \%$ \\
\hline & Tonga & Micha, 2014 (modelling; saturated fat): $22 \cdot 8 \%$ \\
\hline & & Konishi, 2011 (24H-FR; adults 40-59 years): males 33.0 (sD 4.0)\%; females 31.0 (sD 3.0)\% \\
\hline & Vanuatu & Micha, 2014 (modelling; saturated fat): $25 \cdot 7 \%$ \\
\hline \multirow[t]{2}{*}{ Availability } & Samoa & $\begin{array}{l}\text { Martyn, } 2017 \text { (modelling): overall } 96 \mathrm{~g} / \mathrm{d} \text {; Apia } 101 \mathrm{~g} / \mathrm{d} \text {; Northwest Upolu } 87 \mathrm{~g} / \mathrm{d} \text {; Rest of Upolu } \\
\text { 99 g/d; Savaii } 106 \mathrm{~g} / \mathrm{d}\end{array}$ \\
\hline & & Seiden, 2012 (secondary analysis): total fat availability increased by $73 \%$ from 81 to $139 \mathrm{~g} / \mathrm{d}$ \\
\hline \multirow[t]{3}{*}{ Behaviours } & FSM & $\begin{array}{l}\text { Hanson, } 2011 \text { (FFQ; adults): frequency of consumption of turkey tails } 0.8 \mathrm{~d} / \text { week; fried foods } \\
2.5 \mathrm{~d} / \text { week; percentage consuming fried foods (often to daily) } 38.0 \%\end{array}$ \\
\hline & & $\begin{array}{l}\text { Kaufer, } 2010 \text { (FFQ; adult women): frequency of consumption of imported animal fat in } 2005 \\
2.1 \mathrm{~d} / \text { week; in } 20072.4 \mathrm{~d} / \text { week }\end{array}$ \\
\hline & $\begin{array}{l}\text { Solomon } \\
\text { Islands }\end{array}$ & Tsuchiya, 2017 ( $24 \mathrm{H}-\mathrm{FR}$; controls aged $\geq 20$ years): $82.5 \%$ consumed oils and fats daily \\
\hline
\end{tabular}

FSM, Federated States of Micronesia; PNG, Papua New Guinea; RMI, Republic of the Marshall Islands; $24 \mathrm{H}-\mathrm{FR}, 24 \mathrm{~h}$ food recall.

*Values reported as mean total fat intake, unless stated otherwise;

†Values reported as percentage contribution of total fat to energy, unless stated otherwise. 
Table 3 Sugar intake by type of outcome reported and by country

\begin{tabular}{|c|c|c|}
\hline $\begin{array}{l}\text { Type of intake } \\
\text { data }\end{array}$ & $\begin{array}{l}\text { Countries } \\
\text { with data }\end{array}$ & Description of intake \\
\hline Absolute intake & Guam & $\begin{array}{l}\text { Paulino, } 2011 \text { ( } 24 \mathrm{H}-\mathrm{FR} \text {; women } \\
\text { aged } \geq 40 \text { years; added } \\
\text { sugar): feast day } 89.5 \text { (SD } \\
62 \cdot 0) \mathrm{g} / \mathrm{d} \text {; non-feast day } 47.3 \\
(\mathrm{SD} 42 \cdot 2) \mathrm{g} / \mathrm{d}\end{array}$ \\
\hline \multirow[t]{7}{*}{$\begin{array}{l}\text { Household } \\
\text { expenditure }\end{array}$} & $\begin{array}{l}\text { Cook Islands } \\
\text { FSM } \\
\text { Kiribati } \\
\text { Nauru } \\
\text { New } \\
\quad \text { Caledonia }\end{array}$ & $\begin{array}{l}\text { 2007: } 3 \cdot 0 \% \dagger \\
\text { 2014: } 2 \cdot 1 \% \ddagger \\
\text { 2014: } 13 \cdot 0 \% \S \\
\text { 2014: } 5 \cdot 5 \% \| \\
\text { 2009: } 4.7 \% \ddagger ; 0.6 \% \S\end{array}$ \\
\hline & Palau & 2014: 4.0\%‡ \\
\hline & Samoa & 2016: $2 \cdot 2 \% \ddagger$ \\
\hline & $\begin{array}{l}\text { Solomon } \\
\text { Islands }\end{array}$ & $2015,2014: 2 \cdot 6 \% \ddagger ; 2.0 \% \S$ \\
\hline & Tonga & 2010: $4.2 \% \|$ \\
\hline & Tuvalu & 2010: $8.0 \% \not$ \\
\hline & Vanuatu & 2015, 2012: $1.9 \% \S ; 0.04 \% \|$ \\
\hline \multirow{4}{*}{$\begin{array}{l}\text { Percentage } \\
\text { contribution to } \\
\text { energy }^{*}\end{array}$} & Kiribati & $\begin{array}{l}\text { Estime, } 2014 \text { (secondary } \\
\text { analysis): } 34.0 \%\end{array}$ \\
\hline & Samoa & $\begin{array}{l}\text { Martyn, } 2017 \text { (modelling; brown } \\
\text { sugar): } 8.0 \%\end{array}$ \\
\hline & $\begin{array}{l}\text { Solomon } \\
\text { Islands }\end{array}$ & $\begin{array}{l}\text { Estime, } 2014 \text { (secondary } \\
\text { analysis): } 2 \cdot 0 \%\end{array}$ \\
\hline & Vanuatu & $\begin{array}{l}\text { Martyn, } 2015 \text { (modelling): } 3.6 \% \\
\text { Estime, } 2014 \text { (secondary } \\
\text { analysis): } 4.0 \%\end{array}$ \\
\hline \multirow[t]{3}{*}{ Behaviours } & CNMI & $\begin{array}{l}\text { Dela Cruz, } 2016 \text { (questionnaire; } \\
\text { adults aged } \geq 18 \text { years): about } \\
75 \% \text { of adults drink one or } \\
\text { more SSB daily }\end{array}$ \\
\hline & FSM & $\begin{array}{l}\text { Hanson, } 2011 \text { (FFQ; adults): } \\
\text { percentage consuming } \\
\text { imported sugar foods (often to } \\
\text { daily) } 16.0 \% \text {; imported drinks } \\
\text { with sugar (often to daily) } \\
72.0 \%\end{array}$ \\
\hline & & $\begin{array}{l}\text { Kaufer, } 2010 \text { (FFQ; adult } \\
\text { women): frequency of } \\
\text { consumption of sugar } \\
\text { (imported product or added to } \\
\text { local food) in } 2005,3.2 \mathrm{~d} / \text { week; } \\
\text { in } 2007,1.9 \mathrm{~d} / \text { week }\end{array}$ \\
\hline
\end{tabular}

FSM, Federated States of Micronesia; CNMI, Commonwealth of the Northern Mariana Islands; $24 \mathrm{H}-\mathrm{FR}, 24 \mathrm{~h}$ food recall; SSB, sugar-sweetened beverage.

*Values reported as percentage contribution of sugar to energy, unless stated otherwise.

†Confectionery.

¥Sugar, jam, honey, chocolate, confectionery.

$\S$ Sugar alone.

|l Sugar and confectionery.

\section{Housebold expenditure}

Ten countries had household expenditure data on fats and oils, which ranged from $0.01 \%$ in Vanuatu ${ }^{(31)}$ to $3.4 \%$ in Cook Islands ${ }^{(21)}$. On the other hand, eleven countries had expenditure data on sugar although how it was reported varied, with studies reporting on sugar alone $(n 4)$, sugar and confectionery ( $n$ 3), sugar, jam, honey, chocolate and confectionery ( $n$ 6), and one on confectionery alone. Expenditure on sugar, jam, honey, chocolate and confectionery ranged from $2 \cdot 1 \%$ in FSM to $8.0 \%$ in Tuvalu.
Expenditure on sugar alone ranged from $0.6 \%$ in New Caledonia $^{(24)}$ to $13.0 \%$ in Kiribati ${ }^{(44)}$. Only two countries (Cook Islands and Vanuatu) had data on household expenditure on salt $\left(0 \cdot 1^{(21)}\right.$ and $1 \cdot 8 \%{ }^{(31)}$, respectively).

\section{Sources of fat, sugar and salt}

Four countries had data on sources of fat and these were coconut products, oils, meat products and fish products. A study in Tonga ${ }^{(40)}$ reported that imported food products including meats and processed foods contributed $65 \%$ of total daily fat intake. Three countries had data on sources of salt. Bread (15.4\%) was found to be the highest contributor of dietary salt in Vanuatu ${ }^{(43)}$, while table salt was common in Fiji (13.7\%; Fiji National Nutrition Survey 2017, unpublished results) and Samoa $(39 \cdot 0 \%)^{(41)}$. Meat and fish products were consistently among the top sources of salt in these three countries. There were no data on the sources of sugar, but studies in CNMI and FSM reported frequent consumption of sugar-sweetened beverages ${ }^{(32,33)}$.

\section{Percentage contribution to energy intake}

Percentage contribution of total fat to total energy intake varied across the four countries with data $(14.5 \%$ in Solomon Islands ${ }^{(38)}, 23.3 \%$ in $\mathrm{FSM}^{(18)}, 27.5 \%$ in Guam ${ }^{(19)}$, $33 \%$ for males and $31 \%$ for females in Tonga $\left.{ }^{(40)}\right)$. Nine countries estimated percentage contribution of saturated fat to total energy intake from modelling and it ranged from $22 \cdot 8 \%$ in Tonga to $27 \cdot 5 \%$ in Samoa ${ }^{(46)}$. A study in Guam $^{(19)}$ reported the percentage contribution of saturated fat on a regular day $(7.9 \%)$ and a feast day (11.4\%) based on $24 \mathrm{~h}$ food recall. On the other hand, percentage contribution of sugar to total energy intake appeared similar in two countries $3.6 \%$ in Vanuatu ${ }^{(43)}$ and $2 \%$ in Solomon Islands ${ }^{(44)}$ ) but was notably high (34\%) in Kiribati ${ }^{(44)}$. A study in Samoa reported that brown sugar contributed $8 \%$ to total energy intake but did not provide information on total sugar ${ }^{(41)}$.

\section{Availability of fat, sugar and salt for consumption (per capita)}

There was very little information on availability of fat, sugar and salt. In fact, only Samoa had data on this outcome which showed that there was high availability of fats in the country as well as excessive access to sodium ${ }^{(41,42)}$.

\section{Dietary behaviours}

Dietary behaviour data from FSM and Solomon Islands indicated that foods high in fats and oils were consumed frequently ${ }^{(18,33,38)}$. Sugar was also consumed frequently with $75 \%^{(32)}$ and $50 \%^{(33)}$ of adults in CNMI and FSM, respectively, reporting drinking one or more sugarsweetened beverages per day. Studies in Samoa ${ }^{(37)}$ and Vanuatu (Vanuatu Ministry of Health 2017, unpublished results) using the WHO STEPwise approach to surveillance of NCD risk factors (STEPS) instrument ${ }^{(47)}$ reported salt-related behaviours including not adding salt at the 
Table 4 Salt intake by type of outcome reported and by country

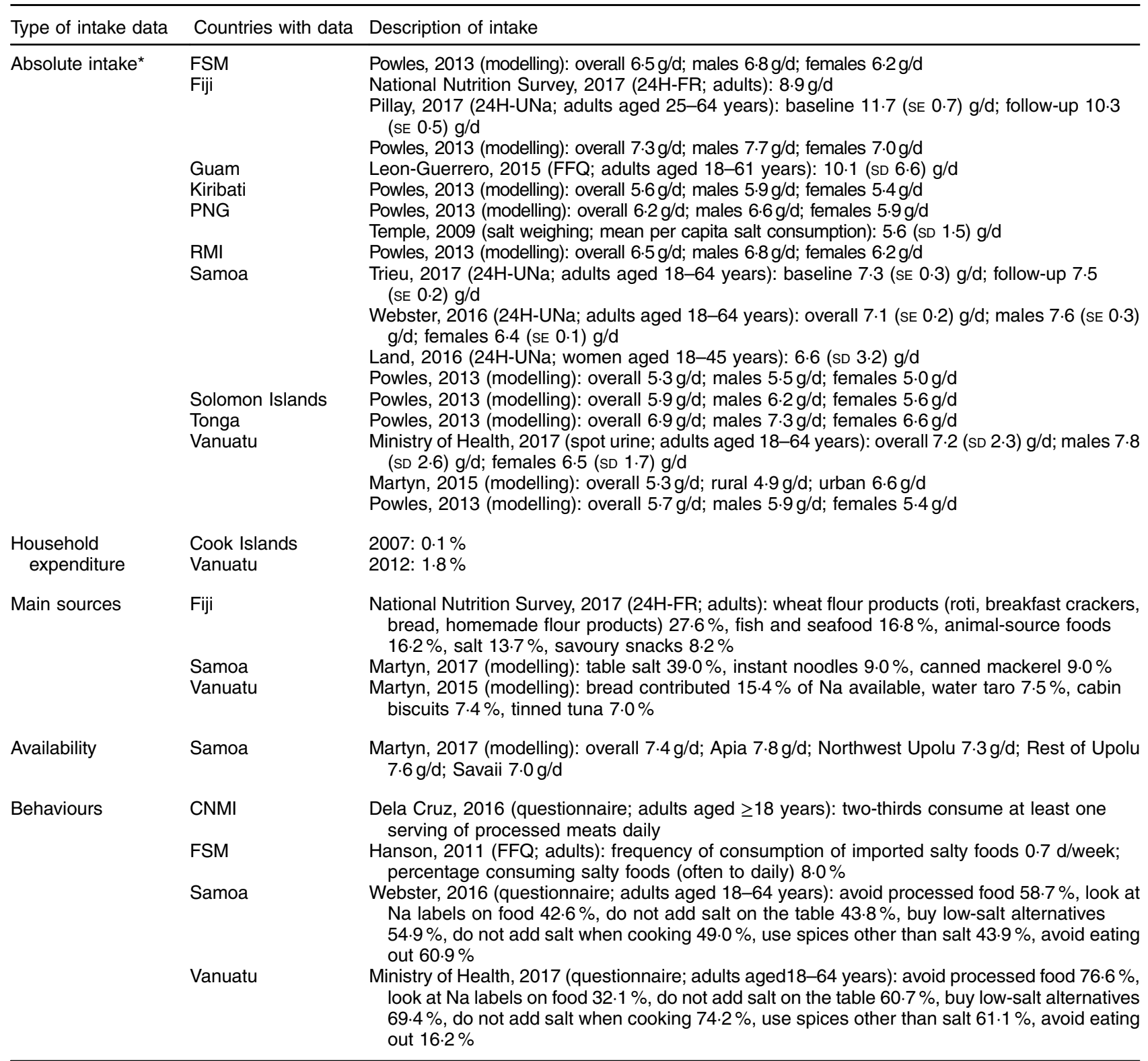

FSM, Federated States of Micronesia; PNG, Papua New Guinea; RMI, Republic of the Marshall Islands; CNMI, Commonwealth of the Northern Mariana Islands; $24 \mathrm{H}-\mathrm{FR}, 24 \mathrm{~h}$ food recall; $24 \mathrm{H}-\mathrm{UNa}$, $24 \mathrm{~h}$ urine collection.

*Values were converted to salt in $\mathrm{g} / \mathrm{d}(393.4 \mathrm{mg}$ sodium $=1 \mathrm{~g}$ salt).

table ( 44 and $61 \%)$, not adding salt when cooking ( 49 and $74 \%$ ), avoiding processed foods (59 and 77\%), looking at $\mathrm{Na}$ labels on foods (43 and $32 \%$ ), buying low-salt alternatives (55 and 69\%), using spices other than salt (44 and $61 \%)$ and avoiding eating out (61 and 16\%).

\section{Discussion}

The current systematic review found that while dietary data were available on fat, sugar and salt intakes in Pacific Island countries, some countries had limited or no data, and most countries lacked high-quality data. In fact, only
Fiji has a recent comprehensive nutrition survey. The absence of quality nutrition data is concerning, given high rates of NCD in the region ${ }^{(1)}$ and changing dietary patterns from traditional diets toward a more Western-style diet as shown by historical trends from previous studies ${ }^{(4,6)}$. Furthermore, changes in local food production, population growth, environmental conditions, increased reliance on processed and imported foods, and lack of policies ${ }^{(12,48)}$ also put Pacific Islanders at a higher risk for NCD. There is a need for better data on Pacific diets to establish benchmark consumption levels, to better inform initiatives to improve diets. Also, quality parameters should be considered when interpreting existing data. 
Table 5 Most recent fat, sugar and salt intake estimates and dietary assessment method used

\begin{tabular}{|c|c|c|c|c|}
\hline Country & Year & & Intake $(g / d)$ & Dietary assessment method \\
\hline \multicolumn{5}{|l|}{ Fat intake } \\
\hline FSM & 2005 & $62 \cdot 6$ & & 24H-FR \\
\hline Fiji & 2017 & $97 \cdot 1$ & $95 \% \mathrm{Cl} 96 \cdot 0,98 \cdot 2$ & 24H-FR \\
\hline Guam & 2015 & 98.9 & SD 67.7 & FFQ \\
\hline Solomon Islands & 2017 & $25 \cdot 4$ & SD $13 \cdot 2$ & $24 \mathrm{H}-\mathrm{FR}$ \\
\hline Tonga & 2011 & $\begin{array}{c}107.0 \mathrm{M} \\
88.0 \mathrm{~F}\end{array}$ & $\begin{array}{l}\text { SD } 21 \cdot 0 \\
\text { SD } 20 \cdot 0\end{array}$ & 24H-FR \\
\hline Vanuatu & 2015 & $75 \cdot 0$ & & Dietary modelling \\
\hline \multicolumn{5}{|l|}{ Sugar intake } \\
\hline Guam & 2011 & $47 \cdot 3$ & SD $42 \cdot 2$ & 24H-FR \\
\hline \multicolumn{5}{|l|}{ Salt intake } \\
\hline FSM & 2013 & $6 \cdot 5$ & $95 \% \mathrm{Cl} 5.5,7.7$ & Dietary modelling \\
\hline Fiji & 2017 & $10 \cdot 3$ & SE 0.5 & $24 \mathrm{H}-\mathrm{UNa}$ \\
\hline Guam & 2015 & $10 \cdot 1$ & SD 6.6 & $\mathrm{FFQ}$ \\
\hline Kiribati & 2013 & $5 \cdot 6$ & $95 \% \mathrm{Cl} 4 \cdot 6,6 \cdot 8$ & Dietary modelling \\
\hline Papua New Guinea & 2013 & $6 \cdot 2$ & $95 \% \mathrm{Cl} 5 \cdot 3,7 \cdot 3$ & Dietary modelling \\
\hline RMI & 2013 & 6.5 & $95 \% \mathrm{Cl} 5.5,7.7$ & Dietary modelling \\
\hline Samoa & 2017 & 7.5 & SE 0.2 & $24 \mathrm{H}-\mathrm{UNa}$ \\
\hline Solomon Islands & 2013 & 5.9 & $95 \% \mathrm{Cl} 5.0,7.0$ & Dietary modelling \\
\hline Tonga & 2013 & $6 \cdot 9$ & $95 \%$ Cl 5.8, $8 \cdot 1$ & Dietary modelling \\
\hline Vanuatu & 2017 & $7 \cdot 2$ & SD $2 \cdot 3$ & Spot urine collection \\
\hline
\end{tabular}

FSM, Federated States of Micronesia; RMI, Republic of the Marshall Islands; M, males; F, females; 24H-FR, 24 hour food recall; $24 \mathrm{H}-\mathrm{UNa}, 24 \mathrm{~h}$ urine collection.

The percentage contribution to total energy intake from fat was below the recommended $30-35 \%$ of total energy $^{(49)}$ for four countries, although in the case of Solomon Islands ${ }^{(38)}$ energy from fat was at the minimum level of $15 \%$ recommended for adequate population consumption of energy ${ }^{(49)}$. Furthermore, it is important to note that absolute intake of total fat was about $100 \mathrm{~g} / \mathrm{d}$ in Fiji, Guam and Tonga, similar to intakes reported in developed countries ${ }^{(50)}$. In terms of saturated fat intake, the modelling study by Micha et al. ${ }^{(46)}$ showed that the nine Pacific Island countries with data had the highest intakes of this nutrient (average of $23.5 \%$ ), far exceeding the recommended limit of $<10 \%$ of total energy ${ }^{(49)}$ and the global average of $9.4 \%$. A review of data from forty countries in 2013 found that total fat intake is correlated with saturated fat intake, but not with polyunsaturated fat intake ${ }^{(51)}$. This suggests that interventions aimed at lowering fat intake should focus not only on lowering saturated fat intake, but also increasing polyunsaturated fat intake ${ }^{(51,52)}$.

There was not enough information about absolute intake of sugar, although based on household expenditure and percentage contribution to energy intake data it appeared that Kiribati had high sugar intakes relative to other Pacific Island countries. Unfortunately, there was no information available about sources of sugar or consumption patterns to further explain this finding, although an earlier study reported that sugar-sweetened beverage consumption in Kiribati was moderate (less than $23 \%$ of 11- to 18-year-olds consuming soft drinks daily and import of less than 40 litres per person ${ }^{(8)}$ ).

All countries with data appeared to be exceeding the WHO recommended limit of less than $5 \mathrm{~g}$ salt/person per day, contributing to the high rates of morbidity and mortality due to $\mathrm{CVD}^{(53)}$. As such, salt reduction initiatives and policies are essential to lower population salt intake and reduce the burden of $\mathrm{NCD}^{(54)}$. A systematic review of salt reduction initiatives around the world in 2015 reported that only a few Pacific Island countries engage in actions to reduce salt intake (e.g. food reformulation and front-ofpack labelling) ${ }^{(55)}$, and a separate review showed that while there has been good progress in terms of planning strategies, barriers such as the lack of capacity to accurately measure salt intake and effectively implement activities hinder further progress ${ }^{(56)}$. The population-based multisectoral salt reduction interventions implemented in Fiji $^{(34)}$ and Samoa ${ }^{(36)}$ both showed a non-significant change in salt intake following the interventions. The authors identified reasons for the null effect such as the short intervention duration (18 months), low intervention dose, lack of participation from other government sectors and limited engagement with the food industry. These suggest the need for stronger and more active mechanisms for multisectoral governance and partnership; engaging with the food industry; and planning, monitoring and evaluation of interventions to reduce salt intake in the region.

Although not specifically covered in the present review, a common theme noted in the identified studies was the increasing reliance of Pacific Islanders on processed foods. This was evident in countries with data on main sources and behaviours, which showed that the consumption of these foods (e.g. canned meat and fish, rice, sugarsweetened drinks and instant noodles, among others) was frequent and common. This trend may also be reflected at feasts. Paulino et al. ${ }^{(19)}$ found that the type of foods served 
during feast days in Guam has shifted from low-energy foods to predominantly high-energy foods such as 'meats, desserts, sodas and other sweetened beverages'. Further studies are warranted to confirm this in other countries. A review of processed foods in Pacific Island countries reported that most are heavily dependent upon food imports with only a small proportion of foods produced locally ${ }^{(48)}$. Previous studies have shown that an increasing reliance on energy-dense nutrient-poor imported foods is associated with increases in $\mathrm{NCD}^{(15,57,58)}$. This demonstrates the wide scope for potential strategies including regulations on imported products, food safety and labelling in the Pacific Islands ${ }^{(48,59)}$.

The current review revealed a range of different dietary assessment methods employed by studies. This variation limits comparison of intakes between countries and over time within a country. Data were available from HIES however, including in five countries where HIES was the only source of nutrition data. In the absence of other national surveys using standardized methodologies, these surveys may provide a good proxy measure of dietary intake ${ }^{(60)}$. If HIES are used in this manner, standardization and reporting of methods may need to be strengthened as most reports did not provide information on standardization methods. We also observed country differences in the way foods were grouped and reported which made them unsuitable for comparison. For instance, sugar intake was reported as sugar alone or confectionery alone, sugar and confectionery combined, or sugar, jam, honey, chocolate and confectionery.

Differences in quality between peer-reviewed studies and grey literature studies were observed. Peer-reviewed studies generally used direct approaches to measuring diet but had poor participation rates, while grey literature studies, which were generally reports of national surveys, had better participation rates but often employed indirect approaches to measure dietary intake. Furthermore, grey literature studies were larger scale (national) compared with peer-reviewed studies which were usually implemented at a community or village level. This suggests that strengthening the quality of nutrition data in the Pacific will require the use of more direct dietary methods in national surveys and efforts to link large- and small-scale studies. This was achieved in Samoa ${ }^{(37)}$ where $24 \mathrm{~h}$ urine collection was measured from a subset of STEPS participants (i.e. targeted 500 individuals out of 2800 STEPS participants) and demonstrated that it was feasible to incorporate salt intake measurements into a populationbased national survey without large additional costs. This is important since the WHO has recommended the integration of salt intake monitoring through urine collection into its STEPS surveys ${ }^{(47)}$.

Strengths of the present review include the systematic approach, the breadth of databases searched, multiple search strategies and extensive grey literature searches, and stakeholder input. All Pacific Island countries were individually searched within the published literature. Quality assessment was conducted at the dietary assessment and study methodology level, which, to the authors' knowledge, has not been done before in a review of dietary intakes of Pacific Island countries. Limitations of the review include the restriction by language and publication date. Given several of the countries included are French-speaking and that all Pacific Island countries have English as a second language, it is likely that nutrition data are reported in other languages that we have missed. Synthesis was also only based on studies that collected data in 2005 and thereafter, and a meta-analysis would have strengthened the analysis but was deemed inappropriate due to the heterogeneity across the studies. Within each type of outcome, it was also not possible to pool the results due to different assessment methods and analytical approaches. The review also focused on adults only. A number of Pacific countries are implementing health promotion programmes in schools, so a similar review of children and adolescents may be helpful.

\section{Conclusions}

In summary, the present study reviews the availability and quality of information on dietary fat, sugar and salt intake in the Pacific Islands. Although varying methods and quality meant that it was difficult to assess and compare studies, the review suggests that intakes of fat, sugar and salt are generally high in countries across the region, with the exception of relatively low salt and fat intakes measured in Solomon Islands. More research of high quality is required throughout the Pacific Islands in order to understand health risks attributable to the diets of its people and inform effective policy interventions.

\section{Acknowledgements}

Acknowledgements: The authors wish to thank Emalie Sparks for her contribution in the technical aspects of the manuscript. Financial support: J.W. is supported by a National Health and Medical Research Council (NHMRC)/ National Heart Foundation Career Development Fellowship (grant number \#1082924) on international strategies to reduce salt. J.W. has funding from WHO, the Victorian Health Promotion Foundation (VicHealth) and NHMRC for research on salt reduction. J.W. is supported through an NHMRC Centre of Research Excellence on food policy interventions to reduce salt (grant number \#1117300). K.T. is supported by an NHMRC postgraduate scholarship (grant number \#1115169) and VicHealth for work on salt reduction. C.B. is a researcher within an NHMRC Centre of Research Excellence in obesity policy and food systems (grant number \#1041020). The funders had no role in the design, analysis, interpretation or writing of this article. 
Conflict of interest: J.W. is Director of the WHO Collaborating Centre on Population Salt Reduction with a remit to support countries to implement and evaluate salt reduction programmes in line with the WHO target for all countries to reduce population salt intake by $30 \%$ by 2025 . All other authors declare no conflicts of interest related to this study. Authorship: J.A.S., J.W., K.T. and B.M. conceived the study. W.S., J.S. and C.B. provided information on available data in Pacific Island countries. J.A.S. and B.M. conducted the risk of bias assessment. J.A.S. wrote the first draft of the paper. All other authors provided comments on the drafts and approved the final paper. Ethics of human subject participation: Not applicable.

\section{Supplementary material}

To view supplementary material for this article, please visit https://doi.org/10.1017/S1368980018003609

\section{References}

1. World Bank (2014) Non-Communicable Disease (NCD) Roadmap Report (English). Washington, DC: World Bank Group.

2. World Health Organization (2011) Global Status Report on Noncommunicable Diseases 2010. Geneva: WHO.

3. Hughes R (2003) Diet, Food Supply and Obesity in the Pacific. Manila: WHO Regional Office for the Western Pacific.

4. Thow AM, Heywood P, Schultz J et al. (2011) Trade and the nutrition transition: strengthening policy for health in the Pacific. Ecol Food Nutr 50, 18-42.

5. Snowdon W \& Thow AM (2013) Trade policy and obesity prevention: challenges and innovation in the Pacific Islands. Obes Rev 14, Suppl. 2, 150-158.

6. Charlton KE, Russell J, Gorman E et al. (2016) Fish, food security and health in Pacific Island countries and territories: a systematic literature review. BMC Public Health 16, 285.

7. World Health Organization (2013) Global Action Plan for the Prevention and Control of Noncommunicable Diseases 2013-2020. Geneva: WHO.

8. McDonald A (2015) Sugar-Sweetened Beverage Tax in Pacific Island Countries and Territories: A Discussion Paper. Noumea, New Caledonia: Secretariat of the Pacific Community.

9. Thow AM, Swinburn B, Colagiuri S et al. (2010) Trade and food policy: case studies from three Pacific Island countries. Food Policy 35, 556-564.

10. McDonald A (2015) What the Pacific \& Mexico can tell us about soft drink taxes and public health. https://blogs. otago.ac.nz/pubhealthexpert/2015/08/18/what-the-pacificmexico-can-tell-us-about-soft-drink-taxes-and-public-health/ (accessed March 2018).

11. World Health Organization (1999) Development of FoodBased Dietary Guidelines for the Western Pacific Region. Manila: WHO Regional Office for the Western Pacific.

12. Iese V, Paeniu L, Pouvalu SIF et al. (2016) Food Security: Best Practices from the Pacific. Suva, Fiji: Pacific Centre for Environment and Sustainable Development (PaCE-SD) and The University of the South Pacific.

13. World Health Organization (2017) Noncommunicable Diseases Progress Monitor, 2017. Geneva: WHO.
14. Tolley H, Snowdon W, Wate J et al. (2016) Monitoring and accountability for the Pacific response to the noncommunicable diseases crisis. BMC Public Health 16, 958.

15. Hughes R \& Lawrence M (2005) Globalisation, food and health in Pacific Island countries. Asia Pac J Clin Nutr 14, 298-305.

16. McGregor A, Bourke RM, Manley M et al. (2009) Pacific Island Food Security: Situation, Challenges and Opportunities. Canberra, ACT: The Australian National University.

17. Whalan S, Farnbach S, Volk L et al. (2017) What do we know about the diets of Aboriginal and Torres Strait Islander peoples in Australia? A systematic literature review. Aust NZ J Public Health 41, 579-584.

18. Kaufer L, Englberger L, Cue R et al. (2010) Evaluation of a 'traditional food for health' intervention in Pohnpei, Federated States of Micronesia. Pacific Health Dialog 16, 61.

19. Paulino YC, Leon Guerrero RT \& Novotny R (2011) Women in Guam consume more calories during feast days than during non-feast days. Micronesica 41, 223-235.

20. Land M-A, Webster JL, Ma G et al. (2016) Salt intake and iodine status of women in Samoa. Asia Pac J Clin Nutr 25, 142-149.

21. Statistics Office, Cook Islands Ministry of Finance and Economic Management (2007) Cook Islands Housebold Expenditure Survey (HIES) 2005-06. Avarua, Rarotonga: Government of the Cook Islands.

22. Statistics Division, Office of Statistics, Budget and Economic Management, Overseas Development Assistance and Compact Management (2014) Federated States of Micronesia Housebold Income and Expenditure Survey 2013/14 Main Analysis Report. Federated States of Micronesia: Government of the Federated States of Micronesia

23. Bureau of Statistics, Department of Finance (2014) Nauru Household Income and Expenditure Survey (HIES) 2012/ 2013 Main Analytical Report. Republic of Nauru: Government of the Republic of Nauru.

24. Baudchon G (2009) Household Consumption in 2008. Noumea, New Caledonia: Institute of Statistics and Economic Studies New Caledonia.

25. Office of Planning and Statistics, Bureau of Budget and Planning, Ministry of Finance (2014) Republic of Palau 2014 Household Income and Expenditure Survey. Melekeok, Republic of Palau: Government of the Republic of Palau.

26. Temple VJ, Oge R, Daphne I et al. (2010) Salt iodization and iodine status among infants and lactating mothers in Papua New Guinea. Afr J Food Agric Nutr Dev 9, 1807-1823.

27. Samoa Bureau of Statistics (2016) Housebold Income and Expenditure Survey 2013/2014 Tabulation Report. Apia, Samoa: Government of Samoa.

28. Solomon Islands National Statistics Office, Ministry of Finance and Treasury (2015) Solomon Islands 2012/13 Household Income and Expenditure Survey National Analytical Report (Volume I). Honiara, Solomon Islands: Solomon Islands Government.

29. Statistics Department (2010) Household Income and Expenditure Survey 2009 Report. Nuku'alofa, Tonga: Government of Tonga.

30. Central Statistics Division (2010) Household Income and Expenditure Survey 2010 Report. Funafuti, Tuvalu: Tuvalu Government.

31. Vanuatu National Statistics Office (2012) Vanuatu Household Income and Expenditure Survey 2010 Report. Port Vila, Vanuatu: Government of Vanuatu.

32. Dela Cruz R \& Cash H (2016) Commonwealth of the Northern Mariana Islands Non-Communicable Diseases and Risk Factor Hybrid Survey Report. Saipan, Northern Mariana Islands: Commonwealth Healthcare Corporation.

33. Hanson M, Englberger L, Duncan B et al. (2011) An evaluation of a nutrition intervention in Kapinga Village on 
Pohnpei, Federated States of Micronesia. Pacific Health Dialog 17, 173.

34. Pillay A, Trieu K, Santos JA et al. (2017) Assessment of a salt reduction intervention on adult population salt intake in Fiji. Nutrients 9, 1350.

35. Leon-Guerrero RT, Chong M, Novotny R et al. (2015) Relative validity and reliability of a quantitative food frequency questionnaire for adults in Guam. Food Nutr Res 59, 26276-26210.

36. Trieu K, Ieremia M, Santos J et al. (2017) Effects of a nationwide strategy to reduce salt intake in Samoa. J Hypertens 36, 188-198.

37. Webster J, Su'a SAF, Ieremia M et al. (2016) Salt intakes, knowledge, and behavior in Samoa: monitoring saltconsumption patterns through the World Health Organization's surveillance of noncommunicable disease risk factors (STEPS). J Clin Hypertens (Greenwich) 18, 884-891.

38. Tsuchiya C, Tagini S, Cafa D et al. (2017) Socioenvironmental and behavioral risk factors associated with obesity in the capital (Honiara), the Solomon Islands; casecontrol study. Obes Med 7, 34-42.

39. Aswani S \& Furusawa T (2007) Do marine protected areas affect human nutrition and health? A comparison between villages in Roviana, Solomon Islands. Coast Manage 35, 545-565.

40. Konishi S, Watanabe C, Umezaki M et al. (2011) Energy and nutrient intake of Tongan adults estimated by 24-hour recall: the importance of local food items. Ecol Food Nutr 50, 337-350.

41. Martyn T, Yi D, Romeo A et al. (2017) Dietary Patterns of Households in Samoa: Identifying the Factors and Food Items Most Important to Understanding Nutrition. Apia, Samoa: FAO.

42. Seiden A, Hawley NL, Schulz D et al. (2012) Long-term trends in food availability, food prices, and obesity in Samoa. Am J Hum Biol 24, 286-295.

43. Martyn T, Yi D \& Fiti L (2015) Identifying the Housebold Factors, and Food Items, Most Important to Nutrition in Vanuatu. Rome: FAO.

44. Estime MS, Lutz B \& Strobel F (2014) Trade as a structural driver of dietary risk factors for noncommunicable diseases in the Pacific: an analysis of household income and expenditure survey data. Global Health 10, 48.

45. Powles J, Fahimi S, Micha R et al. (2013) Global, regional and national sodium intakes in 1990 and 2010: a systematic analysis of $24 \mathrm{~h}$ urinary sodium excretion and dietary surveys worldwide. BMJ Open 3, e003733.

46. Micha R, Khatibzadeh S, Shi P et al. (2014) Global, regional, and national consumption levels of dietary fats and oils in
1990 and 2010: a systematic analysis including 266 countryspecific nutrition surveys. BMJ 348, g2272.

47. World Health Organization (2017) WHO STEPS Surveillance Manual. Geneva: WHO.

48. Snowdon W, Raj A, Reeve E et al. (2013) Processed foods available in the Pacific Islands. Global Health 9, 53.

49. Food and Agriculture Organization of the United Nations (2010) Fats and Fatty Acids in Human Nutrition: Report of an Expert Consultation. FAO Food and Nutrition Paper no. 91. Rome: FAO.

50. Food and Agriculture Organization of the United Nations (2010) Food Consumption: Dietary Fat Consumption. Rome: FAO Statistics Division.

51. Harika RK, Eilander A, Alssema M et al. (2013) Intake of fatty acids in general populations worldwide does not meet dietary recommendations to prevent coronary heart disease: a systematic review of data from 40 countries. Ann Nutr Metab 63, 229-238.

52. Jakobsen MU, O'Reilly EJ, Heitmann BL et al. (2009) Major types of dietary fat and risk of coronary heart disease: a pooled analysis of 11 cohort studies. Am J Clin Nutr 89, 1425-1432.

53. Strazzullo P, D'Elia L, Kandala NB et al. (2009) Salt intake, stroke, and cardiovascular disease: meta-analysis of prospective studies. BMJ 339, b4567.

54. World Health Organization (2016) The SHAKE Technical Package for Salt Reduction. Geneva: WHO.

55. Trieu K, Neal B, Hawkes C et al. (2015) Salt reduction initiatives around the world - a systematic review of progress towards the global target. PLoS One 10, e0130247.

56. Christoforou A, Snowdon W, Laesango N et al. (2015) Progress on salt reduction in the Pacific Islands: from strategies to action. Heart Lung Circ 24, 503-509.

57. Ulijaszek S (2005) Modernisation, migration and nutritional health of Pacific Island populations. Environ Sci 12, 167-176.

58. Brownell KD \& Yach D (2006) Lessons from a small country about the global obesity crisis. Global Health 2, 11.

59. Food Secure Pacific Working Group (2010) Towards a Food Secure Pacific: Framework for Action on Food Security in the Pacific 2011-2015. Port Vila, Vanuatu: Food Secure Pacific Working Group.

60. Smith L, Dupriez O \& Troubat N (2014) Assessment of the Reliability and Relevance of the Food Data Collected in National Housebold Consumption and Expenditure Surveys. International Household Survey Network. 